\title{
Noch wird gewählt
}

Die Zeitungen und das Fernsehen versorgen uns mit unterschiedlichen Meinungen. Die meisten Meinungsäußerungen erfolgen jedoch über soziale Netzwerke. In ihnen gruppieren die Algorithmen Gruppen, die sich ähneln. Kurios ist daran, dass diese programmierten Homogenitäten Sprünge von einer Gruppenzugehörigkeit zur anderen erzeugen. So kommt es ausgehend von Meinungsroutinen zu plötzlichen Veränderungen in öffentlichen Abstimmungen. Briten, die eigentlich stolz auf ihr Demokratieverständnis und auf ihre Stellung in der Europäischen Union sind, stimmen dann für ihre Isolierung durch den Brexit. US-Amerikaner, die so sehr von dem beispielhaften Wert ihres politischen Systems überzeugt sind, und sogar einige Verfechter des Multikulturalismus, wählen Trump. Ehemalige Anhänger der Arbeiterpartei von Lula da Silva und Dilma Rousseff wenden sich jetzt Jair Bolsonaro zu. Welchen Zusammenhang gibt es zwischen dem Verschleiß und der Inkompetenz der Eliten und der schwindenden Rationalität der Wähler?

Zur Beantwortung dieser Frage ist es notwendig, die Mischung aus objektiven und subjektiven Bedingungen zu verstehen, wie zum Beispiel in Brasilien: Trotz großer Enttäuschung gegenüber der Arbeiterpartei (Partido dos Trabalhadores, PT) haben dort $45 \%$ der Wähler der Verlockung durch Bolsonaro widerstanden, und $37 \%$ der Wähler wählten den Kandidaten der PT, der sich im Wahlkampf als Akademiker präsentierte. Wegen solcher Ambivalenzen nehmen einige an, die Demokratie könne sich selbst demokratisieren. Oft werden in diesem Zusammenhang Akteure aufgeführt, die über die Verteidigung der Vielfalt, der Kritik und der Institutionen, die die Macht teilen, noch etwas erreichen: Feministische Bewegungen, Gender-Aktivismus, ethnische Protestbewegungen, Menschenrechtsorganisationen, Gruppen zur Forderung nach einem kostenlosen Zugang zum Internet. Diese Bewegungen treten auch außerhalb der westlichen Hemisphäre auf, was der Annahme derer widersprechen würde, die die Demokratie als rein atlantisches Phänomen, das zum Untergang verurteilt ist, wenn es zu einer Dezentralisierung und Neuordnung globaler Machtstrukturen kommt. Diese Beispiele, die Hoffnung verbreiten sollen, beziehen allesamt soziokulturelle Erfahrungen mit ein, bei denen der Demokratiebegriff nicht zuerst als politisches System verstanden wird, sondern als eine Art der Lebensgestaltung und Form der Entscheidungsfindung.

Selbst an Orten, an denen sich diese Gegenbeispiele in Form von politischen Aktionen konfigurieren - zum Beispiel »YoSoy132« in Mexiko in Op- 
position gegen den Präsidentschaftskandidaten des Partido Revolucionario Institucional (PRI) im Jahr 2012 oder die »Ele Não«-Bewegung gegen Jair Bolsonaro bei den Wahlen 2018 in Brasilien - gehen sie über die Definition von Parteipolitik hinaus. Sie berücksichtigen, wie wir später genauer sehen werden, nicht nur, wer regiert. Vielmehr beziehen diese Bewegungen die Frage ein, wie Genderunterschiede und Unterschiede in der ethnischen Herkunft sowie die mediale Kommunikation, die freie Forschung und die freie Meinungsäußerung in Wissenschaft, Kunst und Kultur gelebt werden können. Die soziokulturelle und kommunikative Agenda ist auch für das Aufkommen heterogener Sektoren relevant, die die neue Rechte bilden: Sie erschweren den Zugang der Bevölkerungsmehrheit zu den Universitäten, zensieren die Presse und verwirren diejenigen, die sich über digitale Netzwerke informieren wollen. Es sind reichlich Motive vorhanden, um zu untersuchen, was mit der Demokratie und den Bürgern im Rahmen neuer kultureller und kommunikationsbezogener Experimente geschieht.

Ein weiterer wichtiger Grund, dem soziokulturellen Verständnis der Bürger einen strategischen Platz einzuräumen, ist, dass weder die politischen Führungspersonen noch die parteipolitischen Eliten bereit zur Selbstkritik sind. Wenn sie Wahlniederlagen erfahren, führen sie diese meist auf feindliche Verschwörungen zurück (»Das ist die Schuld des Imperialismus oder eine Manipulation der Medien in Kooperation mit der Rechten.«). Das mag zwar manchmal eine nützliche Teilerklärung liefern. Wenn Politiker jedoch ihr Fehlverhalten und Korruptionsfälle nicht eingestehen, durch die sie sich selbst in Verruf bringen, machen sie weder ihre scheinheilige Selbstkritik in Form von Wirtschaftsplänen, noch politische Sanktionen gegen angebliche Schuldige glaubhaft. In anderen Fällen werden abstrakte Entitäten verantwortlich gemacht, zum Beispiel »die Bedrohung durch den Weltmarkt«. Doch ihr Handeln und ihre Taktiken in Konfliktsituationen unterminieren ihr selbstprojiziertes Bild als einheitliche, kohärente und allmächtige Akteure.

Die demokratischen Niederlagen und Veränderungen im politischen System werden auch auf die Vorliebe der Wähler für rechte und linke Populisten zurückgeführt. Somit verliert der Begriff Populismus - der in den Sozialwissenschaften niemals eng gefasst oder eindeutig definiert wird - an Bedeutung, sobald das Wählerverhalten auf solch verschiedene Taktikten der Massenkommunikation reduziert wird. Wie kann die Kategorie des Populismus erklärt werden, wenn wir sie auf Berlusconi, Chávez, die Kirchners, Trump, López Obrador und Bolsonaro anwenden? Es ist schwer nachzuvollziehen, wie eine derart diverse Gruppe von Politikern unter einem Begriff zusam- 
mengefasst werden soll. Die Formen der politischen Kommunikation dieser Führungspersönlichkeiten mit den Massen unterscheiden sich erheblich voneinander, während parallel die aktuellen Formen einer auf Algorithmen basierenden Gouvernementalität sich grundsätzlich der staatszentrierten Form vergangener Tage abheben.

Diese »Erklärungen« für das Scheitern der liberalen Demokratie haben eins gemeinsam: Sie schreiben die Zersetzung der liberalen Regime irgendwelchen Eliten oder politischen Führungspersönlichkeiten zu und stellen ihre eigene Rezeption gegenüber dem, was die Bürger denken, fühlen oder tun, kaum in Frage. Die Machthaber zeigen weder Bereitschaft, sich über die groben Umfragetrends und deren Teildiagnosen hinaus zu informieren, noch erkennen sie die globalen Schwächen des Neoliberalismus an. Denn dadurch würde eine Entmystifizierung der ominösen »Märkte« erfolgen. Scheinbar trägt niemand die Verantwortung für die Niederlagen der Sozialdemokraten und der Linken in den letzten Jahren. Um dem Feind sozusagen nicht in die Hände zu spielen, schweigen sie lieber angesichts politischer Katastrophen wie dem Chavismus in Venezuela oder der Präsidentschaft Daniel Ortegas in Nicaragua, sowie zu Korruptionsfällen, die zum Niedergang der progressiven Parteien in den meisten westlichen Ländern beigetragen haben.

Die Gründer des Partido de la Revolución Democrática (PRD) in Mexiko haben gerade wegen solcher Versäumnisse und folgenlosen Fehler aufgegeben. Im Kirchnerismus und im Petismo (für die Arbeiterpartei Brasiliens - PT) führten unter anderem Vertuschungen über abenteuerliche politische deals, die täglich von den Medien aufgedeckt wurden, zum Rücktritt von führenden Politikern und zum Verlust von Millionen von Wählern. Nur sehr wenige Ideologen und Berater dieser selbstzerstörerischen Regierungen nannten im Anschluss an solche Katastrophen einige Ursachen beim Namen:

Lula war ein großartiger Präsident, aber er hat viele Fehler begangen. Er hat das veraltete politische System benutzt, um zusammen mit der Rechten zu regieren. Es gab keinerlei Neuerungen: Weder Steuerreformen, noch Reformen des Systems oder der Massenmedien, und das in einem Moment, in dem seine Partei, die PT, eigentlich über eine große Legitimität verfügte, um diese zu bewerkstelligen. Nach dem alten System zu regieren bedeutete, im Sinne der stolzen Kumpanei und mit weitverbreiteter Korruption in allen Parteien, nicht nur in der PT, zu herrschen. (Martín del Barrio, 2018)

Millionen von Wählern sagten zuvor, dass sie das bereits geahnt hatten. Aus diesem Grund ist es umso wichtiger und interessanter zu verstehen, was mit 
uns Bürgern eigentlich passiert, anstatt endlos auf die vermeintliche Genesung des Parteiensystems oder der Unternehmenspolitik zu hoffen. Es ist zweifelhaft, ob die Demokratie sich selbst demokratisieren kann, wenn sie das Problem nicht selber angeht.

Während ich diesen Abschnitt im Zuge der Vorbereitung der Übersetzung Ende Oktober 2020 nochmal überfliege, erfahre ich, dass 78 \% der Chilenen in einer Volksabstimmung für eine Reformierung der Pinochet-Verfassung gestimmt haben und dass die neue Verfassung nicht vom Kongress, sondern von den Bürgern geschrieben werden soll. Mit dem Wissen, dass hinter diesen 78 \% verschiedene Menschen mit heterogenen Ängsten und Sorgen stehen, stellt sich uns die Frage, was es für die Bürger bedeuten wird, gemeinsam eine neue Verfassung zu schreiben: Wird diese von den alltäglich spürbaren wirtschaftlichen Missständen ausgehen? Von neuen Utopien? Von der Agenda der Frauen (die $50 \%$ der Verfasserschaft ausmachen werden)? Von den Jugendlichen und den Studierenden, die seit einem Jahrzehnt einer der Protagonisten der Proteste sind?

\section{Verbindungen neu denken}

Als wir Bürger erkannten, dass die letzten großen Ideologien - der Marxismus, der Liberalismus und der Neoliberalismus - gescheitert waren, haben wir in der Wissenschaft auch keine neuen, nachhaltigen Vorschläge finden können. Die Philosophie und die Sozialwissenschaften haben in den letzten vier Jahrzehnten folgende simple Metapher vermittelt: Wir verfügen bloß über einen gedanklichen Werkzeugkasten. In diesem Essay sollen diejenigen Bausteine verwendet werden, die auf der Mikro- und Makroebene auf die aktuellen Entwicklungen angewendet werden können. Das Ziel ist eine essayistische Vision im experimentellen Sinne der Wissenschaft, also eine Hypothese - Fehler - Fehlerberichtigung mit offener Reflexion. Das Konzept des Essays nach Adorno ist ebenfalls zentral für diese Analyse, indem es die Komplexität eines Sachverhaltes zeigt, ohne diesen vollständig erklären zu können.

Auch das Nichtvorhandensein integrierter Ansätze ist ein Anlass für die Verflechtung aus Essay und Montage, wie in der Kunst und der Literatur. Dahingehend sollen Teile, beziehungsweise Bruchstücke inklusive Interpretationen vorgestellt werden. Die Koproduktion weitreichender, uneingeschränkter und vielfältiger Perspektiven ist die geteilte Aufgabe zwischen Autor und Lesern, wie wir sie zwischen Künstlern und Schriftstellern und deren Publikum oder Leserschaft kennen. Wir befinden uns sozusagen in einer Erprobung, 